\title{
foxr1 is a novel maternal-effect gene in fish that is required for early embryonic success
}

\author{
Caroline T Cheung $^{1}{ }^{\text {, Amélie Patinote }}{ }^{1}$, Yann Guiguen ${ }^{1}$ ， Julien Bobe ${ }^{\text {Corresp. } 1}$ \\ 1 LPGP, UR1037 Fish Physiology and Genomics, INRA, Rennes, France \\ Corresponding Author: Julien Bobe \\ Email address: Julien.Bobe@inra.fr
}

The family of forkhead box (Fox) transcription factors regulates gonadogenesis and embryogenesis, but the role of foxr 1 in reproduction is unknown. Evolutionary history of foxr1 in vertebrates was examined and the gene was found to exist in most vertebrates, including mammals, ray-finned fish, amphibians, and sauropsids. By quantitative PCR and RNA-seq, we found that foxr 1 had an ovarian-specific expression in zebrafish, a common feature of maternal-effect genes. In addition, it was demonstrated using in situ hybridization that foxr1 was a maternally-inherited transcript that was highly expressed even in early-stage oocytes and accumulated in the developing eggs during oogenesis. We also analyzed the function of foxr 1 in female reproduction using a zebrafish CRISPR/Cas9 knockout model. It was observed that embryos from the foxrl-deficient females had a significantly lower survival rate whereby they either failed to undergo cell division or underwent abnormal division that culminated in growth arrest at around the mid-blastula transition and early death. These mutant-derived eggs contained dramatically increased levels of p21, a cell cycle inhibitor, and reduced rictor, a component of mTOR and regulator of cell survival, which were in line with the observed growth arrest phenotype. Our study shows for the first time that foxrl is an essential maternal-effect gene and may be required for proper cell division and survival via the p21 and mTOR pathways. These novel findings will broaden our knowledge on the functions of specific maternal factors stored in the developing egg and the underlying mechanisms that contribute to reproductive success. 


\section{1 foxr 1 is a novel maternal-effect gene in fish that is required for early}

\section{2 embryonic success}

3

4

5 Caroline T. Cheung ${ }^{(1)}$, Amélie Patinote ${ }^{(1)}$, Yann Guiguen ${ }^{(1)}$, and Julien Bobe ${ }^{(1) *}$

6

7 (1)INRA LPGP UR1037, Campus de Beaulieu, 35042 Rennes, FRANCE.

8

$9 *$ Corresponding author E-mail: julien.bobe@inra.fr

10

11 Short Title: foxrl regulates early embryogenesis

12

13

14 Summary sentence: The foxr 1 gene in zebrafish is a novel maternal-effect gene that is required

15 for proper cell division in the earliest stage of embryonic development possibly as a

16 transcriptional factor for cell cycle progression regulators, $p 21$ and rictor.

17

18 


\section{Abstract}

20 The family of forkhead box (Fox) transcription factors regulates gonadogenesis and

21 embryogenesis, but the role of foxrl in reproduction is unknown. Evolutionary history of foxrl in

22 vertebrates was examined and the gene was found to exist in most vertebrates, including

23 mammals, ray-finned fish, amphibians, and sauropsids. By quantitative PCR and RNA-seq, we

24 found that foxrl had an ovarian-specific expression in zebrafish, a common feature of maternal-

25 effect genes. In addition, it was demonstrated using in situ hybridization that foxr 1 was a

26 maternally-inherited transcript that was highly expressed even in early-stage oocytes and

27 accumulated in the developing eggs during oogenesis. We also analyzed the function of foxr 1 in

28 female reproduction using a zebrafish CRISPR/Cas9 knockout model. It was observed that

29 embryos from the foxrl-deficient females had a significantly lower survival rate whereby they

30 either failed to undergo cell division or underwent abnormal division that culminated in growth

31 arrest at around the mid-blastula transition and early death. These mutant-derived eggs contained

32 dramatically increased levels of $p 21$, a cell cycle inhibitor, and reduced rictor, a component of

33 mTOR and regulator of cell survival, which were in line with the observed growth arrest

34 phenotype. Our study shows for the first time that foxrl is an essential maternal-effect gene and

35 may be required for proper cell division and survival via the $\mathrm{p} 21$ and mTOR pathways. These

36 novel findings will broaden our knowledge on the functions of specific maternal factors stored in

37 the developing egg and the underlying mechanisms that contribute to reproductive success. 
38

39

40

41

42

43

44

45

46

47

48

49

50

51

52

53

54

55

56

57

58

\section{Introduction}

In vertebrates, maternal products including transcripts, proteins, and other biomolecules are necessary for initiating early embryonic development from fertilization until the mid-blastula transition (MBT) when the zygotic genome is activated [1]. Maternal-effect genes are transcribed from the maternal genome and encode the maternal factors that are deposited into the developing oocytes in order to coordinate embryonic development before MBT [2]. We had previously explored the zebrafish egg transcriptome [3] and proteome [4] in order to gain further understanding of the maternal factors that contribute to good quality or developmentally competent eggs that result in high survival of progeny. However, despite the increasing identification of maternal-effect genes and their functions, large gaps still remain especially the role of genes that regulate early embryogenesis.

The forkhead box (Fox) proteins belong to a family of transcription factors that play important roles in cell growth, proliferation, survival, and cell death [5]. Many of these Fox proteins have been shown to be essential to the various processes of embryogenesis. In mammals, knockouts of several fox genes, including foxa 2 , foxo1, and foxf1, result in embryonic lethality due to defects in development of different organs ([5-7]). In reproduction, a recent transcriptomic study in the Nile tilapia, Oreochromis niloticus, showed that more than 50 fox genes were expressed in the gonads, and some of these, including foxl2, foxo 3 , and foxr 1 (formerly known as foxn5), were specific to XX females[8]. foxl2 and its relatives are known to be key players in ovarian differentiation and oogenesis in vertebrates. Fox12 is essential for mammalian ovarian maintenance, and it was demonstrated in Nile tilapia, medaka, and zebrafish that foxl2 is also a critical regulator of ovary development and maintenance [9]. Further, foxo3 was shown to be required for ovarian follicular development, and its knockout in mice led to 
61 sterility in female mutants due to progressive degeneration of the developing oocytes and lack of

62 ovarian reserve of mature oocytes [10]. foxrl was also found to have sexually dimorphic

63 expression in eels (Anguilla anguilla and Monopterus albus) and marine medaka (Oryzias

64 melastigma), and was predominately observed in the ovaries [11-13]. However, despite these

65 observational studies, the function of foxr 1 in vertebrates, especially its role in reproduction,

66 remains unclear. Thus, in this study, we investigated the evolution of foxr 1 and its phylogenetic

67 relationship in a wide range of vertebrate species, as well as its biological function using

68 knockout zebrafish models created by the CRISPR/cas9 system to broaden our knowledge of the

69 evolutionary origin of maternal-effect genes and the underlying mechanisms that contribute to

70 reproductive success in vertebrates.

71

\section{Materials and Methods}

\section{Protein databases}

74 Since our model is based on the zebrafish, all gene/protein nomenclatures will be based on those 75 of fish. First, human (Homo sapiens) forkhead box protein R1 (Foxr1; ENSG00000176302) was 76 used to BLAST for related protein sequences, and the following amino acid data were retrieved

77 from the ENSEMBL database (http://www.ensembl.org/index.html): mouse, Mus musculus; rat,

78 Rattus norvegicus; guinea pig, Cavia porcellus; pig, Sus scrofa; horse, Equus caballus; cow, Bos

79 taurus; panda, Ailuropoda melanoleuca; opossum, Monodelphis domestica; Chinese softshell

80 turtle, Pelodiscus sinensis; armadillo, Dasypus novemcinctus; frog, Xenopus tropicalis (Foxr1 a);

81 fruit fly, Drosophila melanogaster; nematode, Caenorhabditis elegans; sea squirt, Ciona

82 intestinalis; lamprey, Petromyzon marinus; coelacanth, Latimeria chalumnae; spotted gar,

83 Lepisosteus oculatus (Foxr1 a); cod, Gadus morhua (Foxr1 a); fugu, Takifugu rubripes; medaka, 
84 Oryzias latipes (Foxr1 a); platyfish, Xiphophorus maculatus; stickleback, Gasterosteus

85 aculeatus; tetraodon, Tetraodon nigroviridis; tilapia, Oreochromis niloticus; zebrafish, Danio

86 rerio (Foxn1 and Foxn3); and cave fish, Astyanax mexicanus. Then, the protein sequence for

87 zebrafish (Foxr1; NP_001096594.1) was found in the NCBI database

88 (http://www.ncbi.nlm.nih.gov). Using this amino acid sequence as bait in BLAST, the bald 89 eagle, Haliaeetus leucocephalu; penguin, Pygoscelis adeliae; crested ibis, Nipponia nippon; swan goose, Anser cygnoides domesticus; American alligator, Alligator mississippiensis; Chinese

91 alligator, Alligator sinensis; python, Python bivittatus; central bearded dragon, Pogona vitticeps;

92 frog, Xenopus laevis and Xenopus tropicalis (Foxr1 b); medaka, Oryzias latipes (Foxr1 b);

93 northern pike, Esox lucius (Foxr1 a); rainbow trout, Oncorhynchus mykiss (Foxr1 a); coho

94 salmon, Oncorhynchus kisutch; and Atlantic salmon, Salmo salar, protein sequences were 95 extracted and investigated from the NCBI database. Further, using the protein sequence for 96 zebrafish Foxr1, the following protein sequences were extracted from our previously established 97 PhyloFish online database (http://phylofish.sigenae.org/index.html) [14] and analyzed along with 98 the others: spotted gar, Lepisosteus oculatus (Foxr1 b); cod, Gadus morhua (Foxr1 b); bowfin, 99 Amia calva; European eel, Anguilla anguilla; butterflyfish, Pantodon buchholzi; sweetfish, 100 Plecoglossus altivelis; allis shad, Alosa alosa; arowana, Osteoglossum bicirrhosum; panga, 101 Pangasius hypophthalmus; northern pike, Esox lucius (Foxr1 b); eastern mudminnow, Umbra 102 pygmae; American whitefish, Coregonus clupeaformis; brook trout, Salvelinus fontinalis (Foxr1 103 a and b); rainbow trout, Oncorhynchus mykiss (Foxr1 b); European whitefish, Coregonus 104 lavaretus; grayling, Thymallus thymallus; and European perch, Perca fluviatilis. These 105 sequences are compiled in Supplemental Data 1. 


\section{Phylogenetic analysis}

108 The phylogenetic analysis was conducted using the Phylogeny.fr online program $[15,16]$. Amino

109 acid sequences of 73 Foxr1, Foxr2, Foxn1, and Foxn3 proteins from the above-mentioned

110 species were aligned using the MUSCLE pipeline, alignment refinement was performed with

111 Gblocks, and then the phylogenetic tree was generated using the Maximum Likelihood method

112 (PhyML pipeline) with 100 bootstrap replicates.

113

\section{Synteny analyses}

115 Synteny maps of the conserved genomic regions of foxr 1 and foxr 2 were produced with spotted 116 gar as the reference gene using PhyloView on the Genomicus v91.01 website

117 (http://www.genomicus.biologie.ens.fr/genomicus-91.01/cgi-bin/search.pl).

\section{Fish husbandry}

120 Wildtype zebrafish (Danio rerio) of the $\mathrm{AB}$ strain were maintained at $25^{\circ} \mathrm{C}$ in a central filtration

121 recirculating system with a $12 \mathrm{hr}$ light/dark cycle in the INRA LPGP fish facility (Rennes,

122 France). Individual couple pairing was performed by placing a male and a female overnight in a

123 tank with a partition for separation, and in the morning, the divider was removed after which the

124 female released her eggs to be fertilized by the male. All procedures of fish husbandry and

125 sample collection were in accordance with the guidelines set by the French and European

126 regulations on animal welfare. Protocols were approved by the Rennes ethical committee for

127 animal research (CREEA) under approval no. R2012-JB-01.

128 


\section{Quantitative real-time PCR (qPCR)}

130 Tissue samples from 2 wildtype males and 3 wildtype females, and 50-200 fertilized eggs at the

131 one-cell stage from 32 wildtype couplings, which were all used as separate biological replicates,

132 were harvested, and total RNA was extracted using Tri-Reagent (Molecular Research Center,

133 Cincinnati, $\mathrm{OH}$ ) according to the manufacturer's instructions. The tissue and egg samples were

134 flash-frozen in liquid nitrogen immediately upon harvest and stored at $-80^{\circ} \mathrm{C}$ until use. Reverse

135 transcription (RT) was performed using $1 \mu \mathrm{g}$ of RNA from each sample with the Maxima First

136 Strand cDNA Synthesis kit (Thermo Scientific, Waltham, MA). Briefly, RNA was mixed with

137 the kit reagents, and RT performed at $50^{\circ} \mathrm{C}$ for $45 \mathrm{~min}$ followed by a 5 -min termination step at

$13885^{\circ} \mathrm{C}$. Control reactions were run without reverse transcriptase and used as negative control in

139 the qPCR study. qPCR experiments were performed with the Fast-SYBR GREEN fluorophore

140 kit (Applied Biosystems, Foster City, CA) as per the manufacturer's instructions using $200 \mathrm{nM}$

141 of each primer in order to keep PCR efficiency between 90\% and 100\%, and an Applied

142 Biosystems StepOne Plus instrument. RT products, including control reactions, were diluted

$1431 / 25$, and $4 \mu \mathrm{l}$ of each sample were used for each PCR. All qPCR experiments were performed in

144 duplicate using technical replicates. The relative abundance of target cDNA was calculated from

145 a standard curve of serially diluted pooled cDNA and normalized to $18 S, \beta$-actin, and EF $1 \alpha$

146 transcripts. We considered a Ct variation of around 0.5 as acceptable. The $\mathrm{R}^{2}$ values for foxr 1 ,

$147 p 21, p 27$, and rictor were $91.69 \%, 90.57 \%, 90.03 \%$, and 90.36\%, respectively. The primer

148 sequences can be found in Supplemental Data 2. The tissue expression of foxr 1 was detected

149 using the foxr 1 genotyping forward and reverse primers while the mutant form of foxr 1 in the

150 CRISPR/cas9-mutated eggs was assessed with the foxr 1 qPCR forward and reverse primers. 


\section{RNA-seq}

153 Expression profiles in different holostean and teleostean species were obtained using the publicly

154 available PhyloFish database http://phylofish.sigenae.org/index.html. Corresponding RNA-seq

155 data were deposited into Sequence Read Archive (SRA) of NCBI under accession references

156 SRP044781-84, SRP045138, SRP045098-103, and SRP045140-146. The construction of

157 sequencing libraries, data capture and processing, sequence assembly, mapping, and

158 interpretation of read counts were all performed as previously described [14]. The number of

159 mapped reads was then normalized for the foxr 1 gene across the 11 tissues using RPKM

160 normalization.

161

162 In situ hybridization (ISH)

163 Ovary samples were first fixed in 4\% paraformaldehyde overnight, dehydrated by sequential

164 methanol washes, paraffin-embedded, and sectioned to $7 \mu \mathrm{m}$ thickness before being subjected to

165 the protocol. The sections were deparaffinized and incubated with $10 \mu \mathrm{g} / \mathrm{mL}$ of proteinase $\mathrm{K}$ for

1668 minutes at room temperature, followed by blocking with the hybridization buffer ( $50 \%$

167 formamide, $50 \mu \mathrm{g} / \mathrm{mL}$ heparin, $100 \mu \mathrm{g} / \mathrm{mL}$ yeast tRNA, $1 \%$ Tween 20 , and $5 \mathrm{X}$ saline-sodium

168 citrate $[\mathrm{SSC}])$. The probe was diluted to $1 \mathrm{ng} / \mu \mathrm{L}$ in the hybridization buffer and incubated

169 overnight at $55^{\circ} \mathrm{C}$ in a humidification chamber. The probes were synthesized by cloning a

170 fragment of the foxr 1 gene into the pCRII vector using the cloning foxr 1 forward and reverse

171 primers (Supplemental Data 2) and Topo TA Cloning kit (Invitrogen, Carlsbad, CA) as per the

172 manufacturer's protocol. The digoxigenin (DIG)-labeled sense and anti-sense probes were

173 transcribed from Sp6 and T7 transcription sites, respectively, of the vector containing the cloned

174 foxr 1 fragment and purified using $2.5 \mathrm{M} \mathrm{LiCl}$ solution. The purity and integrity of the probes 
175 were verified using the Nanodrop spectrophotometer (Thermo Scientific) and the Agilent RNA

1766000 Nano kit along with the Agilent 2100 bioanalyzer (Santa Clara, CA). The slides were then

177 subjected to 2 washes each with $50 \%$ formamide/2X SSC, $2 \mathrm{X} \mathrm{SSC}$, and $0.2 \mathrm{X} \mathrm{SSC}$ at $55^{\circ} \mathrm{C}$

178 followed by 2 washes with PBS at room temperature. The sections were subsequently blocked

179 with blocking buffer ( $2 \%$ sheep serum, $3 \%$ bovine serum albumin, $0.2 \%$ Tween 20 , and $0.2 \%$

180 Triton-X in PBS), and the anti-DIG antibody conjugated to alkaline phosphatase (Roche

181 Diagnostics, Mannheim, Germany) was diluted by 1/500 and applied for 1.5 hours at room

182 temperature. The sections were washed with PBS and visualized with NBT/BCIP (nitro blue

183 tetrazolium/5-bromo-4-chloro-3-indolyl phosphate).

184

185 CRISPR-cas9 genetic knockout

186 CRISPR/cas9 guide RNA (gRNA) were designed using the ZiFiT [17,18] online software and

187 were made against 2 targets within the gene to generate a genomic deletion of approximately 240

188 base pairs (bp) that spans the last exon which allowed the formation of a non-functional protein.

189 Nucleotide sequences containing the gRNA were ordered, annealed together, and cloned into the

190 DR274 plasmid. In vitro transcription of the gRNA from the T7 initiation site was performed

191 using the Maxiscript T7 kit (Applied Biosystems) and of the cas9 mRNA using the mMESSAGE

192 mMACHINE kit (Ambion/Thermo Scientific) from the Sp6 site, and their purity and integrity

193 were assessed using the Agilent RNA 6000 Nano Assay kit and 2100 Bioanalyzer. Zebrafish

194 embryos at the one-cell stage were micro-injected with approximately 30-40 pg of each

195 CRISPR/cas9 guide along with purified cas9 mRNA. The embryos were allowed to grow to

196 adulthood, and genotyped using fin clip and PCR that detected the area around the deleted

197 region. The full-length wildtype PCR band was $400 \mathrm{bp}$, and the mutant band with the 
198 CRISPR/cas9-generated deletion was approximately 160 bp using the foxr 1 genotyping pair of

199 primers. The PCR bands of the mutants were then sent for sequencing to verify the deletion.

200 Once confirmed, the mutant females were mated with males harboring the vasa::gfp gene, where

201 vasa was fused with $g f p$ at the 3 ' end, to produce F1 embryos, whose phenotypes were

202 subsequently recorded. Images were captured with a Nikon AZ100 microscope and DS-Ri1

203 camera (Tokyo, Japan).

204

205 Genotyping by PCR

206 Fin clips were harvested from animals under anesthesia ( $0.1 \%$ phenoxyethanol) and lysed with

$2075 \%$ chelex containing $100 \mu \mathrm{g}$ of proteinase $\mathrm{K}$ at $55^{\circ} \mathrm{C}$ for $2 \mathrm{hrs}$ and then $99^{\circ} \mathrm{C}$ for 10 minutes.

208 The extracted DNA was subjected to PCR using Jumpstart Taq polymerase (Sigma-Aldrich, St.

209 Louis, MO) and the foxr 1 forward and reverse primers that are listed in Supplemental Data 2.

210

\section{Statistical Analysis}

212 Comparison of two groups was performed using the GraphPad Prism statistical software (La

213 Jolla, CA), and either the Student's t-test or Mann-Whitney U-test was conducted depending on

214 the normality of the groups based on the Anderson-Darling test. A p-value $<0.05$ was considered 215 as significant.

\section{Results}

\section{Phylogenetic analysis of Foxr1-related sequences}

219 foxr 1 and foxr 2 were formerly known as foxn 5 and foxn6, respectively $[19,20]$. To date,

220 there are six reported members of the foxr/foxn family (foxn1, foxn 2, foxn 3, foxn 4 , foxr 1 , and 
221 foxr2). To examine the evolution of foxr 1, we used a Blast search approach using the zebrafish

222 Foxr1 protein sequence as query in various public databases to retrieve 73 protein sequences

223 from other species that could be related to this protein. All retrieved sequences are compiled in

224 Supplemental Data 1. Of note, both Foxr1 and Foxr2 protein sequences were retrieved. In order

225 to verify that the retrieved protein sequences (Supplemental Data 1) were homologous to

226 zebrafish Foxr1, a phylogenetic analysis was performed. Based on the alignment of the retrieved

227 vertebrate Foxr1-related sequences, and using Foxn1 and Foxn3 amino acid sequences as out-

228 groups, a phylogenetic tree was generated (Fig 1). As shown in Fig 1, the common ancestor of

229 the vertebrate foxr 1 and foxr 2 genes diverged from the ancestor of foxn 1 and foxn 3 genes, and

230 these sequences were clearly observed as two separate clades belonging to actinopterygii (ray-

231 finned fish) and sarcopterygii (lobe-finned fish and tetrapods). In addition, Foxr2 was found only

232 in mammals with no homologs detected in actinopterygii as well as sauropsids and amphibians.

233 Remarkably, despite the wide-ranging presence of Foxr1, no related sequences were observed in

234 invertebrates and chondrichthyans (dogfish and sharks) as well as certain species such as chicken

235 (Gallus gallus). On the other hand, several species showed two Foxr1 sequences including the

236 salmonids, rainbow trout (Oncorhynchus mykiss) and brook trout (Salvelinus fontinalis), as well

237 as northern pike (Esox lucius), cod (Gadus morhua), medaka (Oryzias latipes), and spotted gar

238 (Lepisosteus oculatus).

239 Despite the previous report that stated that foxr 2 was absent in tilapia, stickleback,

240 zebrafish, and medaka genomes, we retrieved Foxr2 protein sequences using the zebrafish Foxr1

241 peptide sequence as query. Thus, using zebrafish Foxr1 sequence as the reference protein, we

242 subsequently compared Foxr1 homology with the Foxr1 and Foxr2 sequences from mammals.

243 As shown in Supplemental Data 3, there was 29-37\% identity and 41-53\% similarity between all 
244 sequences, and there did not appear to be any difference in homology between zebrafish Foxr1

245 and mammalian Foxr1 and Foxr2 sequences. Further, there was 47-60\% identity and 59-77\%

246 similarity between mammalian Foxr1 and Foxr2 sequences, indicating that these two proteins are

247 highly similar and probably diverged recently during evolution.

248

Synteny analysis of foxr 1 and foxr 2 genes in vertebrates

250

In order to further understand the origin of the foxr 1 and foxr 2 genes in vertebrates, we

251 performed a synteny analysis of their neighboring genes in representative vertebrate genomes

252 using the basal actinopterygian, spotted gar, as the reference genome and the Genomicus online

253 database (Fig 2). We found that between the spotted gar and mammals, there was conserved

254 synteny of the foxr 1, upk2, ccdc84, rps25, trappc4, slc37a4, and ccdc153 loci in their genomes.

255 In the frog (Xenopus tropicalis) genome, the foxr1, ccdc153, cbl, mcam, and clqtnf5 loci were

256 conserved, while in Coelacanth, foxr1, ccdc84, rps25, trappc4, slc37a4, cbl, ccdc153, mcam,

257 clqtnf5, as well as rnf26 loci were found in the same genomic region as those of the spotted gar.

258 However, amongst the actinopterygians, there was lower conservation of synteny; in zebrafish

259 and cave fish, the foxr $1, \operatorname{ccd} 884$, and mcam loci were conserved while in the other ray-finned fish

260 species, only the foxr 1 loci was found. We further analyzed the foxr 2 sequences that were found

261 only in mammals, and we demonstrate here that they were all presnet on the X chromosome with

262 no apparent conserved synteny of neighboring genes to those found in the spotted gar. Our

263 overall analyses suggest that all the foxr-related sequences that were found were homologs, and

264 the foxr gene in fish species probably derived from the ancestral foxrl gene. Although there was

265 the same degree of protein homology between zebrafish Foxr1 and mammalian Foxr1 and Foxr2

266 sequences, the phylogenetic tree and synteny analyses showed a clear distinction between them, 
267 and the foxr 2 gene probably derived from a later single gene duplication or transposon event as

268 previously suggested [19].

269

270 Expression profiles of foxr1

271 We next focused our efforts on foxrl since it has previously been shown in eel, tilapia,

272 and medaka to be gonad specific and thus may have specific functions in reproduction. In order

273 to investigate the potential functions of foxr 1, we explored its tissue distribution using two

274 different approaches, qPCR and RNA-seq, the latter of which was obtained from the PhyloFish

275 online database [14]. In zebrafish, we observed from both sets of data that foxr 1 mRNA was

276 predominantly expressed in the ovary and unfertilized egg (Fig 3A and 3B). A high expression of

277 foxr 1 transcript was also detected in bone by RNA-seq, but not by qPCR; this could be due to

278 methodological differences as RNA-seq is more sensitive than qPCR since it is designed to

279 detect the sequences all along the transcript while the latter detects just one area of the transcript.

280 Thus, it is possible that a different splice variant of foxrl exists in bone. By ISH, we also

281 demonstrated that foxr 1 transcripts were highly expressed in the ovary in practically all stages of

282 oogenesis (Fig 3C-E; negative controls, Fig 3F-H).

283

284 Functional analysis of foxr 1 in zebrafish

285 To understand the role of foxrl during oogenesis and early development, we performed

286 functional analysis by genetic knockout using the CRISPR/cas9 system. One-cell staged embryos

287 were injected with the CRISPR/cas9 guides that targeted foxr1 and allowed to grow to

288 adulthood. Mosaic founder mutant females (F0) were identified by fin clip genotyping and

289 subsequently mated with vasa::gfp males, and embryonic development of the F1 fertilized eggs 
290 was recorded. Since the mutagenesis efficiency of the CRISPR/cas9 system was very high, as

291 previously described [21,22], the foxr 1 gene was sufficiently knocked-out even in the mutant

292 mosaic F0 females. This was evidenced by the substantially lower transcript level of foxr 1 in the

293 F1 embryos as compared to those from control pairings ( $0.78 \pm 0.22$ and $1.65 \pm 0.04$, respectively;

294 Fig 4A). Thus, the phenotypes of foxr $1(\mathrm{n}=5)$ mutants could be observed even in the F0

295 generation. Since none of the mutated genes were transmissible to future generations neither

296 through the male nor the female (ie. all the surviving embryos were WT), therefore, all of our

297 observations were obtained from the F1 generation, which were the fertilized eggs derived from

298 the mosaic female foxr 1 mutants.

299 We observed that most of the embryos from the foxr 1 mutant females had a very low

300 developmental success at $24 \mathrm{hpf}(23.2 \pm 8.4 \%$ vs. $85.2 \pm 9.2 \%$ in controls; $\mathrm{p}<0.008)$ (Fig 4B). The

301 frequency of the mutation in the mutant females is demonstrated in Fig 4C, and it was observed

302 that 3 of the mutants produced abundant non-developing eggs that remained non-cellularized,

303 reflecting their failure to undergo cell division (Fig 5E-H). The eggs derived from these 3 foxr 1

304 mutant females did not undergo any cell division at $2 \mathrm{hpf}$ and continued to display a complete

305 lack of development up to $8 \mathrm{hpf}$. By $24 \mathrm{hpf}$, these non-developing eggs that failed to divide were

306 all dead. In addition, two of the mutants produced developmentally incompetent eggs with two

307 phenotypes; those with a non-cellularized morphology (Fig 5E-H), and another population that

308 developed albeit with an abnormal morphology (Fig 5I-L). These fertilized and developing

309 embryos were structurally abnormal, with unsmooth and irregularly-shaped yolk as well as

310 asymmetrical cell division that culminated into a blastodisc with a group of cells on top of an

311 enlarged syncytium (Fig 5K, arrow). These eggs underwent developmental arrest at around $4 \mathrm{hpf}$ 
312 or the MBT and appeared to regress with further expansion of the syncytium (Fig 5J-K) until 313 death by $24 \mathrm{hpf}$.

314 The observed phenotype of the foxr 1 mutant-derived uncellularized eggs was very similar 315 to previously described unfertilized eggs [23]. Thus, the foxR1 mutant females were mated with $316 v a s a:: g f p$ males, and the genotype of their progeny was assessed for the presence of the $g f p$ gene, 317 which would only be transmitted from the father since the mutant females did not carry this 318 gene. We found that these uncellularized eggs from the foxr 1 mutant females did indeed carry 319 the gfp gene (Fig 5M), which indicated that some or all of them were fertilized, but were arrested 320 from the earliest stage of development and did not undergo any cell division. These novel 321 findings showed for the first time that foxr 1 is essential for the developmental competence of 322 zebrafish eggs, and is therefore a crucial maternal-effect gene.

323 In order to delve into the possible mechanisms that may be involved in the reduced

324 reproductive success of the foxr 1 mutants, we investigated the expression levels of $p 21, p 27$, and 325 rictor, which were previously reported to be repressed by the Foxr1 transcription factor in mice 326 (Santo et al, 2012). We found that there was substantially increased expression of $p 21$ (4.82 \pm 1.09 327 vs $0.25 \pm 0.04$ in controls; $\mathrm{p}<0.002)$ while that of rictor was significantly decreased $(0.87 \pm 0.13$ vs $3281.60 \pm 0.25$ in controls; $\mathrm{p}<0.01)$ in the foxr 1 mutant-derived eggs as compared to eggs produced 329 by wildtype females (Fig 6A-C). The expression of p27 was unchanged between the two groups. 330 These results were in line with a growth arrested phenotype that was observed in the 331 uncellularized and developmentally challenged eggs from the foxr 1 mutant females.

\section{Discussion}


In this study, we first investigated the evolutionary history of foxrl in order to gain

336 perspective into its phylogenetic relationship among homologs from a wide range of species and

337 to clarify its origins. Using the zebrafish protein sequence as query to search for homologs in

338 other species, we retrieved Foxr1 sequences from a broad variety of vertebrates, including

339 actinopterygii, sarcopterygii, and sauropsids which suggested the importance of this protein in

340 most vertebrates. We also retrieved Foxr2 sequences from many vertebrates due to its high

341 similarity to the zebrafish Foxr1 peptide (Supplemental Data 3), although we and others

342 demonstrated that the foxr 2 gene is absent from all actinopterygii and sauropsid species, and can

343 only be found in mammals. Evidence from the phylogenetic analyses showed a clear distinction

344 in derivation of the actinopterygian foxr 1 and the mammalian foxr 2 ; the divergence of the

345 ancestral foxr 1 gene in actinopterygii from that of the sarcopterygii and sauropsids occurred

346 quite early in evolution, while the divergence of mammalian foxr 1 and foxr 2 is a much more

347 recent event (Fig1). Further, the synteny analysis (Fig2) showed that there was much

348 conservation of genomic synteny surrounding the foxrl loci between the basal actinopterygian,

349 spotted gar, and actinopterygii and sauropsids, while the neighboring loci around the foxr 2 were

350 completely different in comparison to those next to foxr 1 which suggested that foxr 2 originated

351 from a recent gene duplication or transposition event as previously proposed [19]. We also found

352 that in a small subset of species [rainbow trout (Oncorhynchus mykiss) and brook trout

353 (Salvelinus fontinalis), as well as northern pike (Esox lucius), cod (Gadus morhua), medaka

354 (Oryzias latipes), and spotted gar (Lepisosteus oculatus)], two Foxr1 sequences were observed.

355 This suggested that independent gene duplication events occurred in these lineages. It is also

356 possible that foxr 1 was duplicated in the ancestral actinopterygii followed by the loss of 1 copy

357 in a lineage-dependent manner. Finally, it appeared that the different whole-genome duplication 
358 events, the teleost-specific genome duplication (TGD) and salmonid-specific genome duplication

359 (SaGD) did not impact the current foxr 1 gene diversity because in most species, only one foxr 1

360 gene was retained. The presence of two foxrl sequences in the above-mentioned species could

361 also be due to independent and phylum-specific gene retention or independent gene duplication

362 events that occurred only in these species or technical differences due to different sequencing

363 procedures. Further phylogenetic, synteny, and functional analyses on the two copies of foxr 1 in

364 these species are warranted in order to verify the functionality of both genes.

365 The essentialness of foxr 1 was suggested by the wide-ranging presence of this gene in

366 most vertebrates and the retention of a single copy in most teleosts despite multiple whole

367 genome duplication events, but its biological function is still largely unknown. Previous reports

368 have demonstrated the predominant expression of foxr 1 mRNA in the ovary of medaka, eel, and

369 tilapia $[8,11,13]$, but it was found mostly in the male germ cells and spermatids in mouse and

370 human [24]. It was further shown to be abundantly expressed in the early cleavage and gastrula

371 stages of Xenopus embryos, but absent in post-gastrula stages due to rapid degradation of its

372 mRNA, indicating that it is a maternally-inherited transcript [25]. Thus, the foxrl gene may play

373 different roles in reproduction in teleost fish/amphibians and mammals, suggesting that foxr2 in

374 mammals may have evolved to have comparable functions to the teleost/amphibian foxrl while

375 mammalian foxr 1 is mostly involved in male reproduction and development. Of note, the

376 mammalian foxr 2 is thus far observed only on the $\mathrm{X}$ chromosome highly indicating a function in

377 female reproduction (Fig 2). Future studies to test this are necessary to confirm the function of

378 foxr2. To confirm these results found in other teleosts in zebrafish, we first examined the

379 expression profile of foxr 1 in various tissues, and we showed by qPCR as well as by RNA-seq

380 that there was also an ovarian-specific expression of foxr 1 and negligible amount in the testis as 
381 in the other fish species. By ISH, we found that the foxr 1 transcript was progressively stored in

382 the growing oocytes from the very early stages (Fig 3C-D, arrows) to later staged oocytes (Fig

383 3D-E), and could be found abundantly in mature fertilized eggs (Fig 3B and Fig 4A). These

384 results demonstrated that foxr 1 is one of the maternal products that is deposited into the

385 developing oocytes during oogenesis in zebrafish as observed in other fish species. These

386 findings suggest that foxr 1 may function as a maternal-effect gene such as $n p m 2 a, n p m 2 b$ [26],

387 bucky ball [27], futile cycle [28], and wnt [29].

388 In recent years, there has been an increasing identification of maternal-effect genes and

389 their functions, but there is still limited information on the role of genes that regulate early

390 embryogenesis. Previous studies have found that irreducible, indivisible, atomos, cellular island,

391 cellular atoll, and nebel genes among others have roles in early embryonic cell cleavage and cell

392 division, but their protein identity as well as their regulatory mechanisms are largely unknown or

393 not yet clarified [30,31]. Having established that foxr 1 was indeed a maternal factor, we

394 investigated its function via mutagenic analysis with CRISPR/cas9. We used the F0 mosaic

395 mutant females that were shown to have a decreased level of foxr 1 mRNA for analysis due to the

396 difficulty in transmitting the mutated foxr 1 gene to future generations as both the F0 foxr 1

397 mutant females and males produced mostly non-viable progeny, and the surviving descendants

398 were all of wildtype genotype. We found that the foxrl mutant females produced bad quality

399 eggs, and the developmental success of their progeny was very low, similar to that of foxl2 and

400 foxo3 mutants. These latter two genes have been previously shown to be crucial in ovarian

401 determination since they are necessary for the development of the ovary as well as ovarian fate

402 maintenance via suppression of male cues $[9,10]$. Deficiency in either of these two genes results

403 in small ovaries and disorganized follicles that cannot mature as well as the appearance of testis- 
404 specific cells. Thus, it is possible that foxr 1 may be also required for proper ovarian development 405 and function, as also observed for foxl2 and foxo 3 mutants, and further histological analysis on 406 ovaries from the foxr 1 mutants is warranted. In addition, we found that the foxr 1 mutant-derived 407 eggs were non-cellularized and did not undergo subsequent cell division despite being fertilized, 408 as also observed for eggs derived from $n p m 2 b$ mutant females [26]. This suggested that their 409 defect did not lie in the capability to be fertilized, as seen in slc29ala and otulina mutants [3], 410 but in the cell cycle and proliferation processes. Thus, we investigated the expression profiles of $411 p 21, p 27$, and rictor, which are all cell cycle and cell survival regulators, since Santo et al had 412 previously knocked down foxrl using short hairpin RNAs in mammalian cells and found it to be 413 a transcriptional repressor of them [32]. In this report, we also observed a dramatic increase in 414 p21 transcript in the eggs from foxr 1 mutant females, although the expression of $p 27$ was 415 unchanged, while that of rictor was decreased. Both $p 21$ and $p 27$ are well known cell cycle 416 inhibitors, and rictor is a component of the mTOR (mammalian target of rapamycin) complex 417 that is a major regulator of cell growth and proliferation [33,34]. In fact, mitogens or some 418 survival signal activates a survival cascade, such as the PI3K/Akt pathway, which is activated by 419 the rictor-mTOR complex and promotes cell growth through repression of the negative cell cycle 420 modulators, including $p 21$ and $p 27$ [35]. Thus, our findings were in line with a phenotype of 421 growth arrest and anti-proliferative effects as seen in our eggs derived from foxr 1 mutant 422 females. The different results that we observed as compared to those from Santo et al were 423 probably due to species- and cell type-specific effects.

424 In this study, we showed that foxr 1 is found in a wide-range of vertebrates and is 425 homologous to the foxr 1 genes found in other species. In teleosts, foxr 1 expression is found 426 predominately in the ovary while in mammals, it appears to be specific to the male germline 
427 [24]. We also found that foxrl is a novel maternal-effect gene and is highly expressed in the

428 developing oocytes as well as accumulated in mature eggs to be used in early embryogenesis.

429 Our findings suggest that maternally-inherited foxr 1 may be required for the first few cleavages

430 after fertilization for proper cell growth and proliferation possibly via $p 21$ and rictor, since

431 deficiency in foxr 1 leads to either complete lack of or abnormal cell division culminating to early

432 death in the fertilized egg. Further molecular analyses to disrupt $p 21$ and rictor expression in

433 foxr 1 mutants to investigate their function in these mutants are necessary. Thus, the results of

434 this study are the first to establish a link between egg quality, the control of early cell cycle, and

435 the molecular regulatory mechanisms via the potential transcriptional regulator, FoxR1.

436

437 Conclusions

438 Our study shows for the first time that foxrl is an essential maternal-effect gene and is 439 required for proper cell division and survival possibly via the p21 and mTOR pathways. These

440 novel findings will broaden our knowledge on the functions of specific maternal factors stored in

441 the developing egg and the underlying mechanisms that contribute to reproductive fitness. 


\section{Acknowledgements}

443 The authors would like to thank all the members of the LPGP laboratory for their assistance. We

444 thank JJ Lareyre for the use of the vasa::gfp males for the fertilization assays.

445

\section{References}

447 [1] Baroux C, Autran D, Gillmor CS, Grimanelli D, Grossniklaus U. The maternal to zygotic 448 transition in animals and plants. Cold Spring Harb Symp Quant Biol 2008; 73:89-100.

449 [2] Lindeman RE, Pelegri F. Vertebrate maternal-effect genes: Insights into fertilization, early 450 cleavage divisions, and germ cell determinant localization from studies in the zebrafish.

$451 \quad$ Mol Reprod Dev 2010; 77:299-313.

452 [3] Cheung CT, Nguyen T, Le Cam A, Patinote A, Journot L, Reynes C, Bobe J. Lost in 453 translation: egg transcriptome reveals molecular signature to predict developmental $454 \quad$ success and novel maternal-effect genes. BioRxiv 2018.

455 [4] Yilmaz O, Patinote A, Thao T, Nguyen V, Com E, Lavigne R, Pineau C, Sullivan C V, 456 Bobe J. Scrambled eggs : Proteomic portraits and novel biomarkers of egg quality in 457 zebrafish (Danio rerio). PLoS One 2017; 12:1-24.

458 [5] Hannenhalli S, Kaestner KH. The evolution of Fox genes and their role in development 459 and disease. Nat Rev Genet 2009; 10:233-240.

460 [6] Martins R, Lithgow GJ, Link W. Long live FOXO: Unraveling the role of FOXO proteins 461 in aging and longevity. Aging Cell 2016; 15:196-207.

462 [7] Mahlapuu M, Ormestad M, Enerback S, Carlsson P. The forkhead transcription factor 463 Foxf1 is required for differentiation of extra-embryonic and lateral plate mesoderm.

464 Development 2001; 128:155-166. 
465 [8] Yuan J, Tao W, Cheng Y, Huang B, Wang D. Genome-wide identification, phylogeny, 466 and gonadal expression of fox genes in Nile tilapia, Oreochromis niloticus. Fish Physiol

467 Biochem 2014; 40:1239-1252.

468 [9] Bertho S, Pasquier J, Pan Q, Le Trionnaire G, Bobe J, Postlethwait JH, Pailhoux E, 469 Schartl M, Herpin A, Guiguen Y. Fox12 and Its Relatives Are Evolutionary Conserved Players in Gonadal Sex Differentiation. Sex Dev 2016; 10:111-129.

471

[10] Hosaka T, Biggs WH, Tieu D, Boyer AD, Varki NM, Cavenee WK, Arden KC.

472 Disruption of forkhead transcription factor (FOXO) family members in mice reveals their functional diversification. Proc Natl Acad Sci U S A 2004; 101:2975-80.

474 [11] Geffroy B, Guilbaud F, Amilhat E, Beaulaton L, Vignon M, Huchet E, Rives J, Bobe J, 475 Fostier A, Guiguen Y, Bardonnet A. Sexually dimorphic gene expressions in eels: useful markers for early sex assessment in a conservation context. Sci Rep 2016; 6:34041.

477

478

479

480

481

482

483

484

485

486

487

[12] Chi W, Gao Y, Hu Q, Guo W, Li D. Genome-wide analysis of brain and gonad transcripts reveals changes of key sex reversal-related genes expression and signaling pathways in three stages of Monopterus albus. PLoS One 2017; 12:1-19.

[13] Lai KP o., Li JW, Wang SY ua., Chiu JM a. Y, Tse A, Lau K, Lok S, Au DW a. T, Tse WK a. F, Wong CK on. C, Chan TF, Kong RY ue. C, et al. Tissue-specific transcriptome assemblies of the marine medaka Oryzias melastigma and comparative analysis with the freshwater medaka Oryzias latipes. BMC Genomics 2015; 16:135.

[14] Pasquier J, Cabau C, Nguyen T, Jouanno E, Severac D, Braasch I, Journot L, Pontarotti P, Klopp C, Postlethwait JH, Guiguen Y, Bobe J. Gene evolution and gene expression after whole genome duplication in fish: the PhyloFish database. BMC Genomics 2016; 17:368. [15] Dereeper A, Guignon V, Blanc G, Audic S, Buffet S, Chevenet F, Dufayard JF, Guindon 
S, Lefort V, Lescot M, Claverie JM, Gascuel O. Phylogeny.fr: robust phylogenetic analysis for the non-specialist. Nucleic Acids Res 2008; 36:465-469.

490 [16] Dereeper A, Audic S, Claverie JM, Blanc G. BLAST-EXPLORER helps you build 491 datasets for phylogenetic analysis. Evol Biol 2010; 10:8-13.

492 [17] Sander JD, Maeder ML, Reyon D, Voytas DF, Joung JK, Dobbs D. ZiFiT (Zinc Finger 493 Targeter): an updated zinc finger engineering tool. Nucleic Acids Res 2010; 38:W462-8.

494 [18] Mali P, Yang L, Esvelt KM, Aach J, Guell M, Dicarlo JE, Norville JE, Church GM. RNA495 496 Guided Human Genome Engineering via Cas9 2013; 823:823-827.

498

[20] Katoh M, Katoh M. Identification and characterization of human FOXN6, mouse Foxn6, 499 and rat Foxn6 genes in silico. Int J Oncol 2004; 25:219-223.

500 [21] Auer TO, Duroure K, De Cian A, Concordet JP, Del Bene F. Highly efficient

501 CRISPR/Cas9-mediated knock-in in zebrafish by homology-independent DNA repair. Genome Res 2014; 24:142-153.

503 [22] Gagnon JA, Valen E, Thyme SB, Huang P, Akhmetova L, Pauli A, Montague TG, 504 505 506 Zimmerman S, Richter C, Schier AF. Efficient mutagenesis by Cas9 protein-mediated oligonucleotide insertion and large-scale assessment of single-guide RNAs. PLoS One 2014; 9:e98186.

507 [23] Dekens MP, Pelegri FJ, Maischein HM, Nusslein-Volhard C. The maternal-effect gene 508 futile cycle is essential for pronuclear congression and mitotic spindle assembly in the 509 zebrafish zygote. Development 2003; 130:3907-3916.

510 [24] Petit FG, Kervarrec C, Jamin SP, Smagulova F, Hao C, Becker E, Jégou B, Chalmel F, 
511 Primig M. Combining RNA and Protein Profiling Data with Network Interactions

512 Identifies Genes Associated with Spermatogenesis in Mouse and Human1. Biol Reprod

$513 \quad 2015 ; 92: 1-18$.

514 [25] Schuff M, Rossner A, Donow C, Knochel W. Temporal and spatial expression patterns of 515 FoxN genes in Xenopus laevis embryos. Int J Dev Biol 2006; 50:429-434.

516 [26] Cheung CT, Pasquier J, Bouleau A, Nguyen T-V, Chesnel F, Guiguen Y, Bobe J. Double

517 Maternal Effect: Duplicated Nucleoplasmin 2 Genes, npm2a And npm2b, Are Shared By

518 Fish And Tetrapods, And Have Distinct And Essential Roles In Early Embryogenesis.

$519 \quad$ BioRxiv 2017.

520 [27] Bontems F, Stein A, Marlow F, Lyautey J, Gupta T, Mullins MC, Dosch R. Bucky Ball 521 Organizes Germ Plasm Assembly in Zebrafish. Curr Biol 2009; 19:414-422.

522 [28] Lindeman RE, Pelegri F. Localized products of futile cycle/lrmp promote centrosome523 nucleus attachment in the zebrafish zygote. Curr Biol 2012; 22:843-851.

524 [29] Nicol B, Guiguen Y. Expression profiling of Wnt signaling genes during gonadal 525 differentiation and gametogenesis in rainbow trout. Sex Dev 2011; 5:318-329.

526 [30] Dosch R, Wagner DS, Mintzer KA, Runke G, Wiemelt AP, Mullins MC. Maternal control 527 of vertebrate development before the midblastula transition: mutants from the zebrafish I. 528 Dev Cell 2004; 6:771-780.

529 [31] Pelegri F, Knaut H, Maischein H-M, Schulte-Merker S, Nüsslein-Volhard C. A mutation 530 in the zebrafish maternal-effect gene nebel affects furrow formation and vasa RNA localization. Curr Biol 1999; 9:1431-1440.

532 [32] Santo EE, Ebus ME, Koster J, Schulte JH, Lakeman A, Van Sluis P, Vermeulen J, 533 Gisselsson D, Øra I, Lindner S, Buckley PG, Stallings RL, Vandesompele J, Eggert A, 
534 Caron HN, Versteeg R, Molenaar JJ. Oncogenic activation of FOXR1 by 11q23

535 intrachromosomal deletion-fusions in neuroblastoma. Oncogene 2012;31:1571-1581.

536 [33] Tateishi Y, Matsumoto A, Kanie T, Hara E, Nakayama K, Nakayama KI. Development of

537 mice without Cip/Kip CDK inhibitors. Biochem Biophys Res Commun 2012; 427:285-

2938.

539 [34] Peponi E, Drakos E, Reyes G, Leventaki V, Rassidakis GZ, Medeiros LJ. Activation of 540 mammalian target of rapamycin signaling promotes cell cycle progression and protects 541 cells from apoptosis in mantle cell lymphoma. Am J Pathol 2006; 169:2171-2180.

542 [35] Sarbassov DD, Ali SM, Sabatini DM. Growing roles for the mTOR pathway. Curr Opin $543 \quad$ Cell Biol 2005; 17:596-603.

544 [36] Kimmel CB, Ballard WW, Kimmel SR, Ullmann B, Schilling TF. Stages of embryonic 545 development of the zebrafish. Dev Dyn 1995; 203:253-310. 
Figure 1 (on next page)

Phylogenetic tree of vertebrate Foxr1 and Foxr2 proteins.

This phylogenetic tree was constructed based on the amino acid sequences of Foxr1 proteins (for the references of each sequence see Supplemental Data 1) using the Maximum Likelihood method with 100 bootstrap replicates. The number shown at each branch node indicates the bootstrap value (\%). The tree was rooted using Foxn1 and Foxn3 sequences. The Foxr1 sequences are in red, Foxr2 sequences are in blue, those of Foxn1 are in green, and Foxn3 sequences are in purple. $a$ and $b$ denote multiple copies of Foxr1 sequences in fish. 


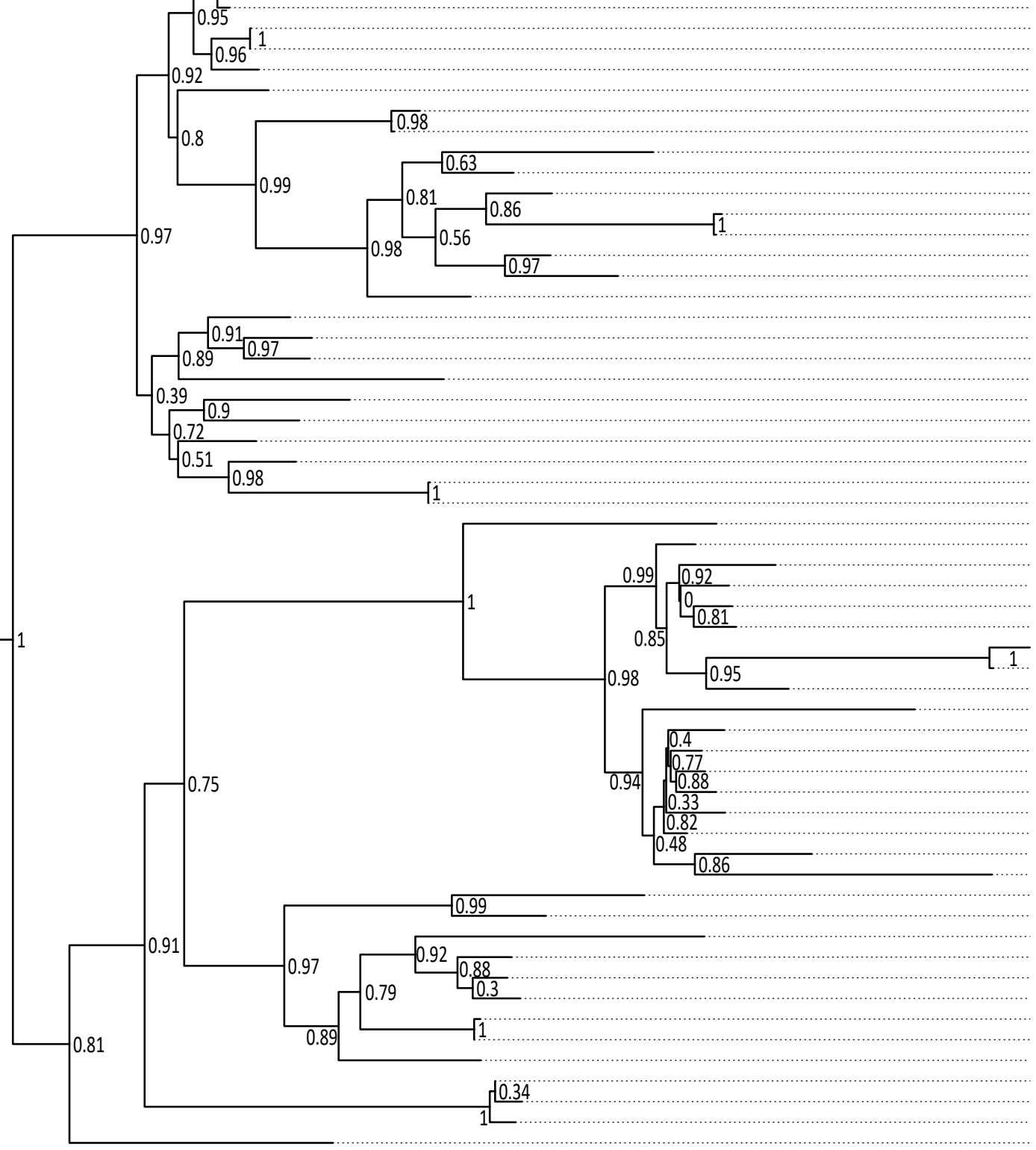

odf a

Stickleback

European Perch

Medaka a

Fusu

Tetraodon

Platyfish

Zebrafish

Panga

Cave fish

Allis Shad

Arowana
Butterflytish

European Eel

Spotted

Spotted Gar a

Spotted Gar

Armadillo

Horse
Panda

Pig

Cow

Mouse

Human
Armadillo

Panda1

pig

Human

Mouse

Horse

Rat

Guinea Pig

Central Bearded Drago Python Swan Goose Penguin

Crested Ibis Bald Eagle
American Alligator Chinese Alligator Chinese Sottshell Turtle Xenopus tropical
Xenopus laevis Xenopus tropicalis b Coelacanth Lamprey Sea Squirt Zebratish Human Fruitfly-jumu 
Figure 2 (on next page)

Conserved genomic synteny of foxr1 genes

Genomic synteny maps comparing the orthologs of foxr1, foxr2, and their neighboring genes, which were named after their human orthologs according to the Human Genome Naming Consortium (HGNC). Orthologs of each gene are shown in the same color, and the chromosomal location is shown next to the species name. foxrl orthologs are boxed in red while foxr2 orthologs are boxed in blue. Light grey boxes denote the lack of synteny of the corresponding genes in the indicated species as compared to gar. 


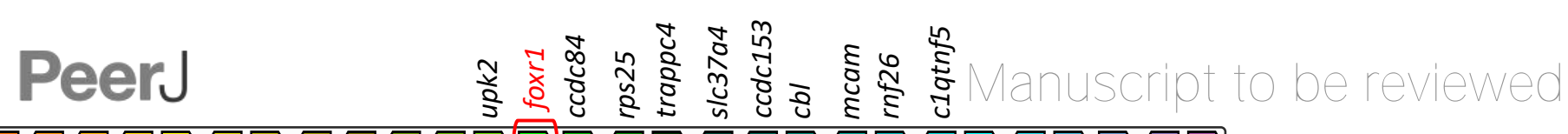

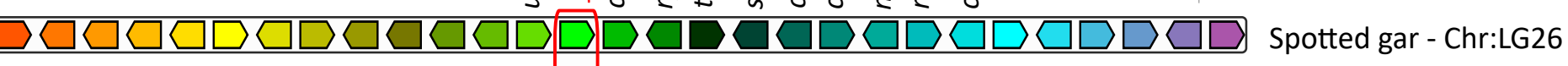



\begin{tabular}{|c|c|c|}
\hline \multicolumn{2}{|c|}{ foxrz } & \\
\hline  &  & Cow - Chr:X \\
\hline 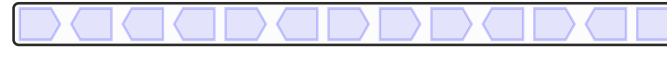 & 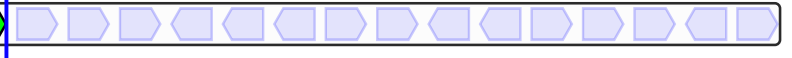 & Sheep - Chr: \\
\hline$\langle\langle\langle\rangle \mid \square\rangle \square$ & 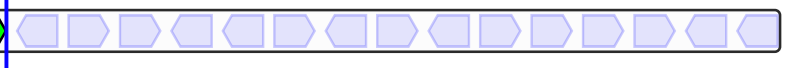 & Pig - Chr:X \\
\hline$\frac{a b}{\square}$ & 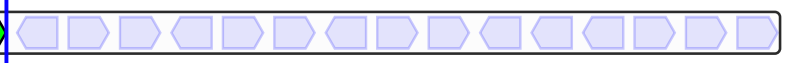 & - Chr:X \\
\hline 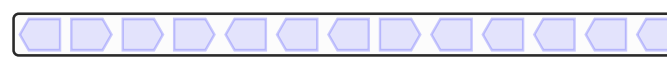 & 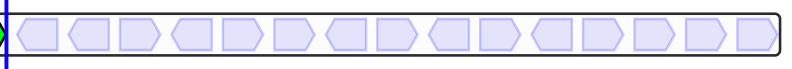 & Dog - Chr:X \\
\hline  & 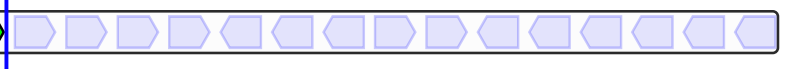 & \\
\hline$\square \square \square \square<a \square \square \square$ & 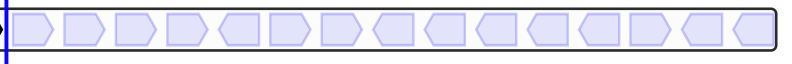 & $\mathrm{Chr}$ \\
\hline$\square \square \backslash$ Peer) reviewing PDF $1(20$ & $2018) \square \square D \square \square \square \square D$ & - Chr:X \\
\hline & & \\
\hline
\end{tabular}




\section{Figure 3 (on next page)}

Expression profile of foxr1 in zebrafish

Tissue expression analysis of foxr1 mRNA in adult zebrafish (A) by quantitative real-time PCR (qPCR) and (B) RNA-seq. Expression level by qPCR is expressed as a normalized value following normalization using 18S, $\beta$-actin, and ef1 $\alpha$ expression while that by RNA-seq is expressed in read per kilobase per million reads (RPKM). Tissues were harvested from 3 to 4 wildtype zebrafish individuals. (C-H) In situ hybridization was performed for foxr1 in zebrafish ovaries from wildtype females. Positive staining is demonstrated using the antisense probe against foxr1 (Fig 3C-E) in blue with 5-bromo-4-chloro-3-indolyl-phosphate/nitro blue tetrazolium as substrate. The negative control was performed with the sense probe (Fig 3F-H). 20X magnification; scale bar denotes $90 \mu \mathrm{m} . \mathrm{N}=5$ each for foxr 1 mutant and control. UF, unfertilized. 
PeerJ

A

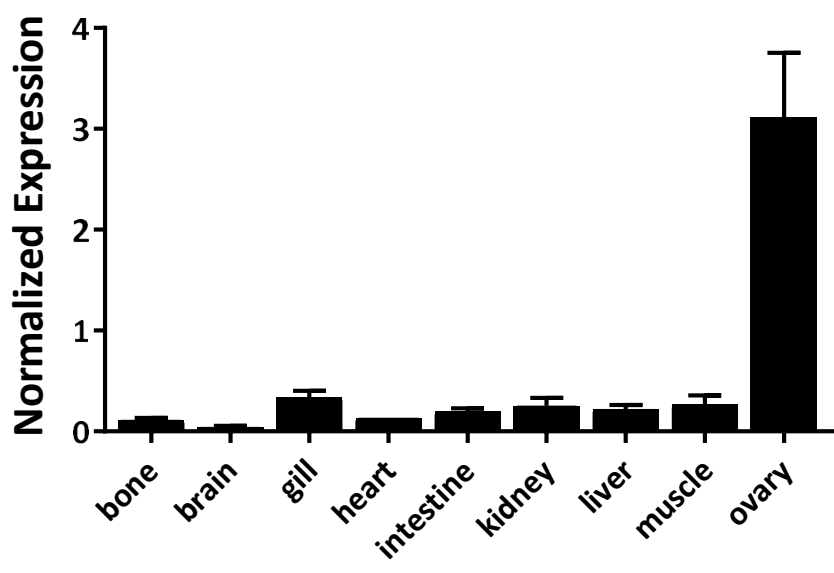

B



\section{Anti-sense}
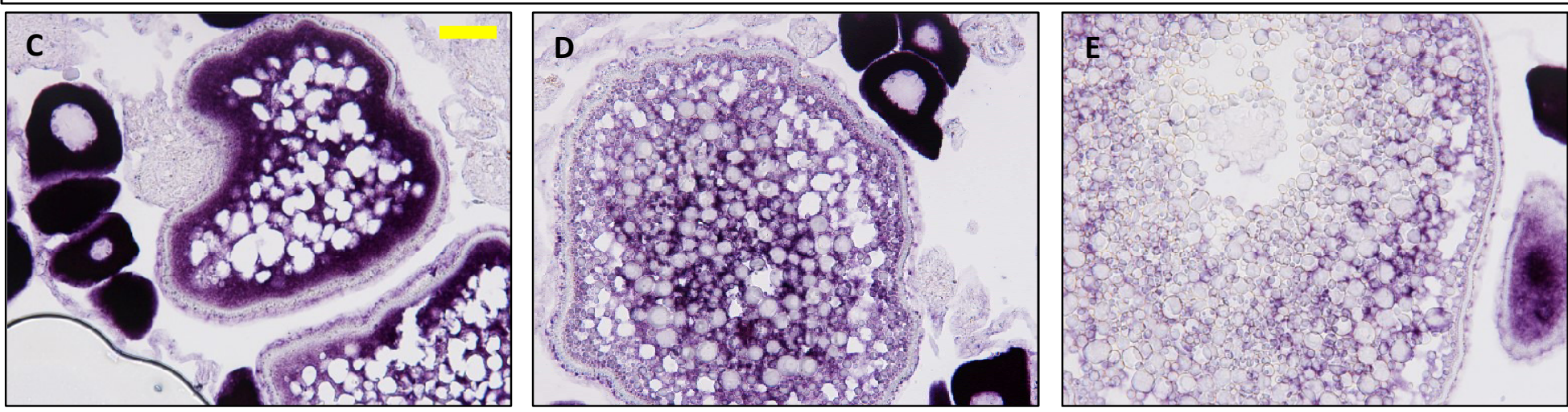

Sense controls
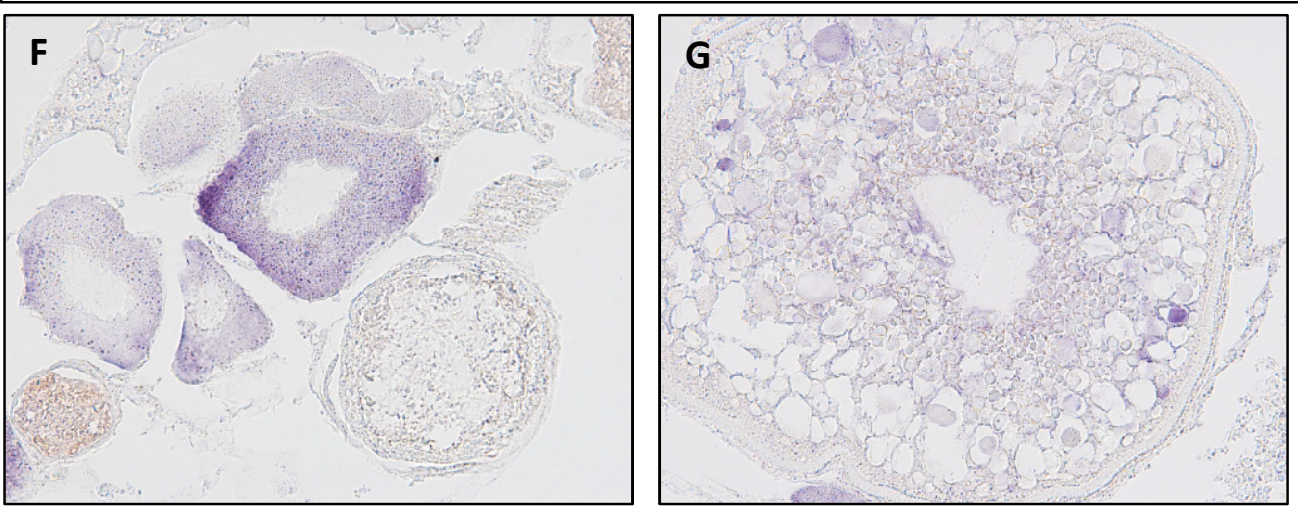

H 


\section{Figure 4(on next page)}

CRISPR/cas9 knockout of foxr1 in zebrafish

(A) Normalized expression level of foxr1 transcript by quantitative real-time PCR (qPCR) in the fertilized zebrafish eggs from crosses between foxr1 mutant females and vasa::gfp males. (B) Developmental success (\% survival) at 24 hours post-fertilization (hpf) as measured by the proportion of fertilized eggs that underwent normal cell division and reached normal developmental milestones based on Kimmel et al. [36] from crosses between foxr1 mutant females and vasa::gfp males. (C) Frequency of foxr1 mutant phenotypes in the F1 eggs between crosses of foxr1 mutant females and vasa::gfp males. "Embryos did not develop at all (please refer to Fig 5E-H). ${ }^{+}$Embryos had a partially cellularized blastodisc that was sitting atop an enlarged syncytium (please refer to Fig 5I-L). The graphs demonstrate representative data from a single clutch from a mutant female. qPCR data were normalized to $18 S, \beta$-actin, and ef1 $\alpha . \mathrm{N}=5$ each for foxr 1 mutant and control. All assessments were performed from at least 3 clutches from each mutant. ** $p<0.01$ by Mann-Whitney U-test. Control = eggs from crosses of wildtype females with vasa::gfp males; foxr1 = eggs from crosses of foxr1 mutant females with vasa::gfp males. 
PeerJ

A

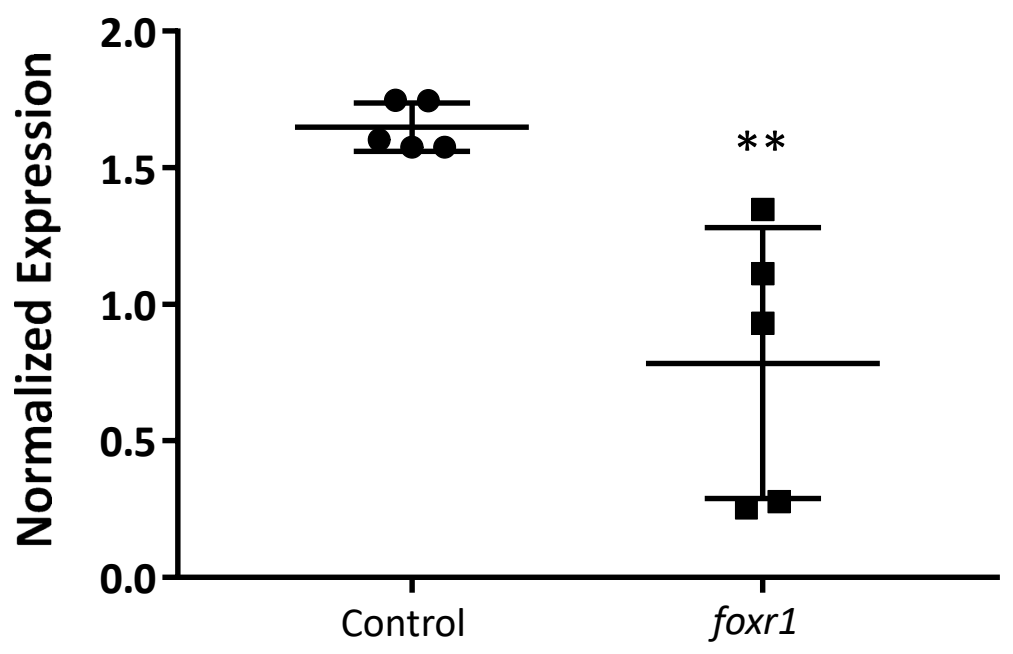

Manuscript to be reviewed

B

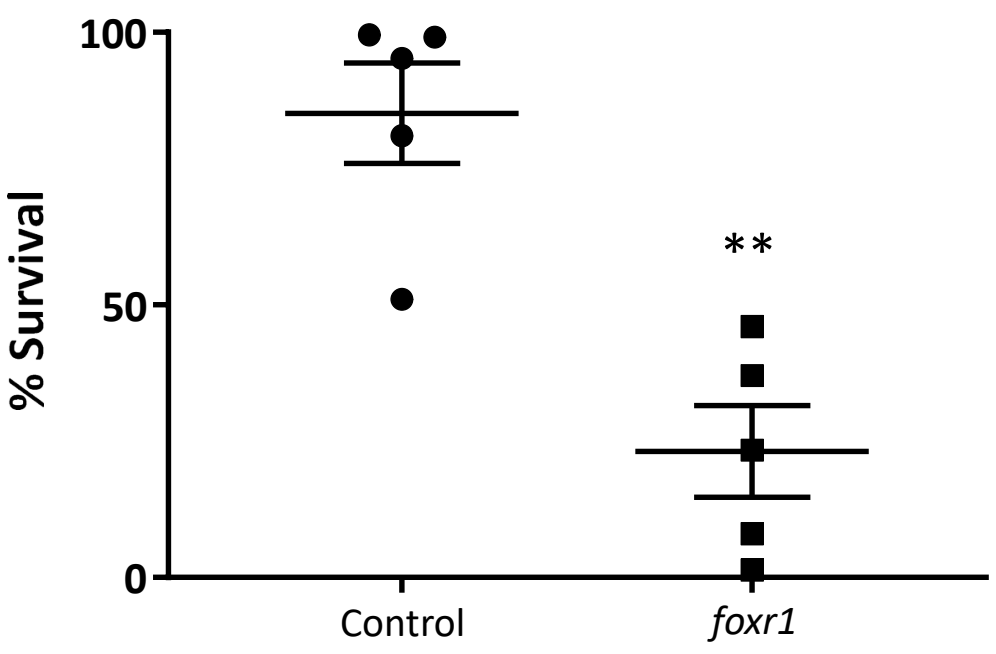

C

Frequency of foxr1 mutant phenotypes

\begin{tabular}{|c|c|c|c|c|c|}
\hline \multirow{2}{*}{$\begin{array}{c}\text { Individual } \\
\text { mutant }\end{array}$} & $\begin{array}{c}\text { Total number } \\
\text { of embryos }\end{array}$ & $\begin{array}{c}\text { Embryos with defects } \\
\text { cellularized }\end{array}$ & $\begin{array}{c}\text { Non- } \\
\text { Cellularized with } \\
\text { developmental } \\
\text { defects }\end{array}$ & $\begin{array}{c}\text { Normal } \\
\text { embryos }\end{array}$ & $\begin{array}{c}\text { Survival } \\
\text { of normal } \\
\text { embryos to } \\
\text { total } \\
\text { embryos }\end{array}$ \\
\cline { 2 - 4 } foxr1-1 & 517 & 333 & 0 & 184 & $36 \%$ \\
foxr1-2 & 183 & 183 & 0 & 0 & $0 \%$ \\
foxr1-3 & 212 & 195 & 0 & 17 & $8 \%$ \\
foxr1-4 & 435 & 194 & 60 & 201 & $46 \%$ \\
foxr1-5 & 268 & 84 & 80 & 104 & $39 \%$ \\
WT-1 & 478 & 91 & 0 & 387 & $81 \%$ \\
WT-2 & 297 & 3 & 0 & 294 & $99 \%$ \\
WT-3 & 223 & 11 & 0 & 212 & $95 \%$ \\
WT-4 & 256 & 56 & 0 & 200 & $78 \%$ \\
WT-5 & 196 & 2 & 0 & 194 & $99 \%$ \\
\hline
\end{tabular}




\section{Figure 5 (on next page)}

Effect of foxr1 deficiency on zebrafish embryogenesis

Representative images demonstrating development of fertilized eggs from crosses between control (A-D) and foxr1 (E-L) females and vasa::gfp males from 2-24 hours post-fertilization (hpf). In the control eggs, the embryos were at 64-cell (A), oblong (B), germ ring (C), and 24-somite (D) stages according to Kimmel et al [36] . Eggs from foxr1 mutant females were non-developing with a non-cellularized morphology (E-H) or developing with an abnormal morphology (I-L). (A, E, I) = images taken at 2 hpf; (B, F, J) = images taken at 4 hpf; (C, G, $\mathbf{K})=$ images taken at $6 \mathrm{hpf} ;(\mathbf{D}, \mathbf{H}, \mathbf{L})=$ images taken at $24 \mathrm{hpf}$. Scale bar denotes $500 \mu \mathrm{m}$. The arrow demonstrates an abnormally cellularized blastodisc that was sitting atop an enlarged syncytium. (M) Genotypic analysis of the eggs from crosses of foxr1 mutant females and vasa::gfp males to determine fertilization status. The gfp and vasa primers produced a band that was 1333 base pairs in size. Detection of the $n p m 2 b$ gene (band size $=$ 850 base pairs) was used as a control. Con = eggs from crosses of wildtype females with vasa::gfp males; foxr1 = eggs from crosses of foxr1 mutant females with vasa::gfp males. $\mathrm{N}=5$ each for foxr 1 mutant and control. 
Figure 6 (on next page)

Expression profiles of $p 21, p 27$, and rictor in eggs from foxr1 mutant females

Fertilized eggs from foxr1 mutant females were subjected to qPCR for examination of the transcript levels of $p 21, p 27$, and rictor. The graphs demonstrate representative data from a single clutch from a mutant female. Data were normalized to $18 S, \beta$-actin, and ef1 $\alpha . \mathrm{N}=4$ each for foxr1 mutant and control, at least two clutches were used from each animal, and each experiment was performed in duplicate. ${ }^{*} p<0.05,{ }^{*} p<0.01$ by Mann-Whitney U-test. 
PeerJ

A

p21

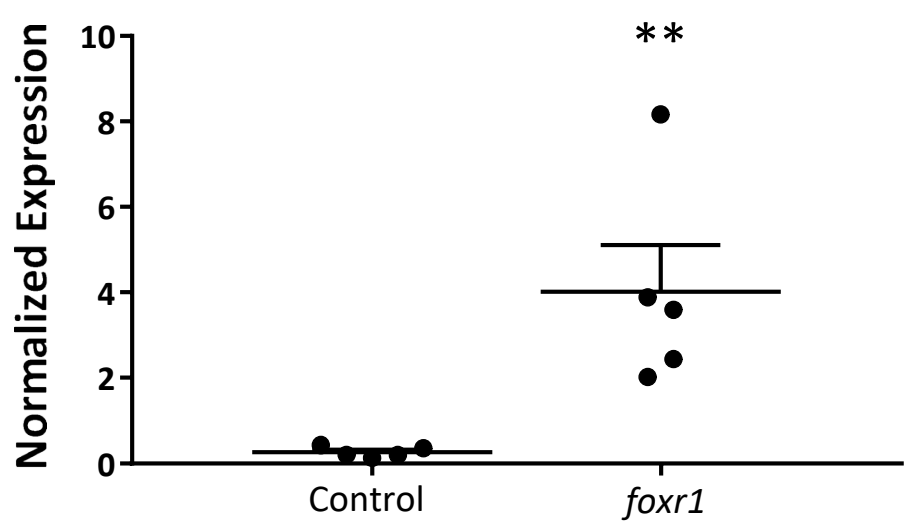

C

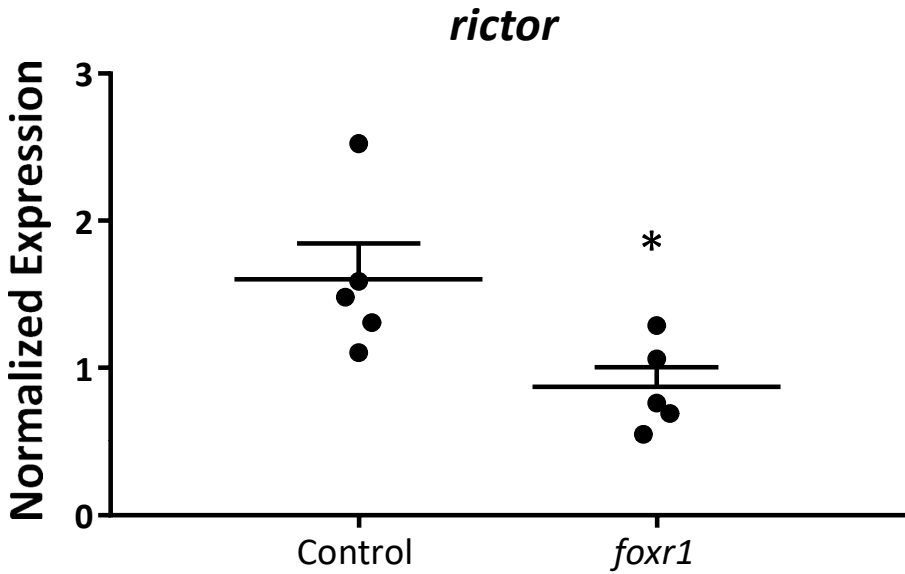

Manuscript to be reviewed

B

p27

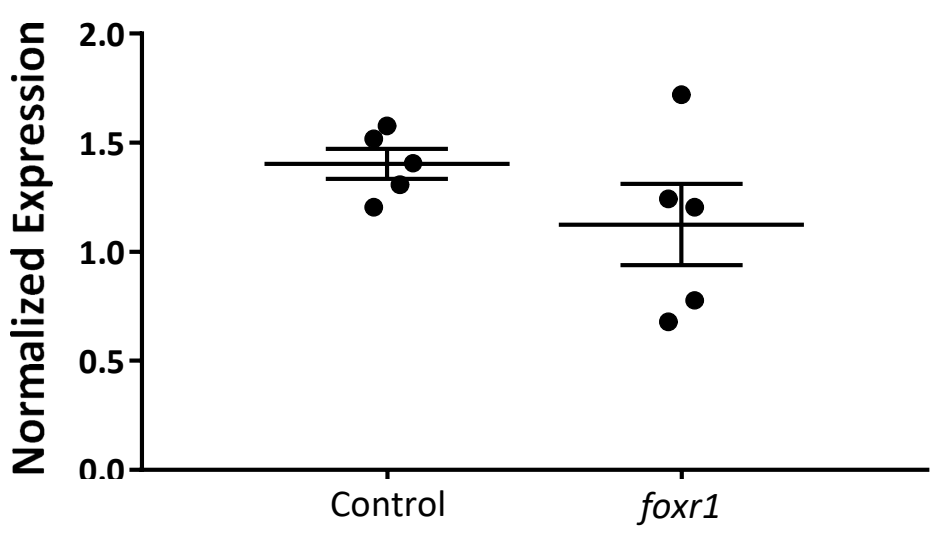

